\title{
Calretinin, CD34, and $\alpha$-smooth muscle actin in the identification of peritoneal invasive implants of serous borderline tumors of the ovary
}

\author{
Eung Seok Lee ${ }^{1}$, Anthony S-Y Leong ${ }^{2}$, Young-Sik Kim ${ }^{1}$, Ju-Han Lee ${ }^{1}$, Insun Kim³ \\ Geung Hwan $\mathrm{Ahn}^{4}$, Hye Sun $\mathrm{Kim}^{5}$ and Yi Kyeong Chun ${ }^{5}$
}

${ }^{1}$ Department of Pathology, Ansan Hospital, Korea University, Ansan, Gyeonggi-Do, Korea; ${ }^{2}$ Division of Anatomical Pathology, Hunter Area Pathology Service and Discipline of Anatomical Pathology, University of Newcastle, NSW, Australia; ${ }^{3}$ Department of Pathology, Guro Hospital, Korea University, Seoul, Korea; ${ }^{4}$ Department of Pathology, Samsung Medical Center, Sungkyunkwan University, School of Medicine, Seoul, Korea and ${ }^{5}$ Department of Pathology, Samsung Cheil Hospital, Sungkyunkwan University, School of

Medicine, Seoul, Korea

\begin{abstract}
The correct identification of invasive implants in the peritoneum in serous borderline tumors (SBTs) of the ovary is an important determinant of diagnosis, treatment, and prognosis. Although the histologic criteria to distinguish noninvasive from invasive implants have been defined, the distinction can still be difficult. We examined the presence and distribution of mesothelial cells, stromal fibrocytes, and myofibroblasts in invasive and noninvasive peritoneal implants in 100 noninvasive, 100 invasive, and 100 metastatic nests/foci from 20 cases of SBTs with peritoneal implants, 10 serous carcinomas with peritoneal metastasis, and 10 cases of endosalpingiosis by immunostaining for calretinin, CD34, and $\alpha$-SMA. All 100 invasive nests from seven SBTs and all 100 metastatic nests from the cases of serous carcinoma showed loss of calretinin + mesothelial cells and stromal CD34 + fibrocytes around the nests. In contrast, 72/100 noninvasive nests displayed the presence of mesothelial cells around the nests and 68 displayed preservation of surrounding stromal fibrocytes. $\alpha$ smooth muscle actin positive myofibroblasts were present as a stromal response in 100/100 metastatic nests, $100 / 100$ invasive nests and 54/100 noninvasive nests. The loss of mesothelial cells and stromal fibrocytes surrounding invasive nests together with a proliferation of myofibroblasts as demonstrated by immunostaining proved to be a sensitive and specific tool to separate invasive from noninvasive implants and represents an important adjunct to morphologic diagnosis. Combined sensitivity and specificity of the three antibodies was 100 and $81 \%$, respectively. These methods, however, may not be helpful for small biopsies of noninvasive desmoplastic implants. The distribution of these cells provides some insights into the histogenesis of invasive and noninvasive implants in SBTs.

Modern Pathology (2006) 19, 364-372. doi:10.1038/modpathol.3800539; published online 13 January 2006
\end{abstract}

Keywords: immunohistochemistry; invasive implants; ovarian serous borderline tumors

Serous borderline tumor (SBT) of ovary was formally recognized as an entity by the International Federation of Gynecology and Obstetrics (FIGO) and the World Health Organization (WHO) in the early 1970s. ${ }^{1,2}$ Stage is a powerful predictor of outcome, and recurrence is more frequently associated with

Correspondence: Dr ES Lee, MD, Department of Pathology, Ansan Hospital of Korea University, 516 Bunsi, Gojan-Dong, Danwon-Gu, Ansan, Gyeonggi-Do 425-707, South Korea.

E-mail: a9604@chollian.net

Received 14 September 2005; revised 15 November 2005; accepted 16 November 2005; published online 13 January 2006 high-stage disease. The presence of invasive peritoneal implants is the best predictor of outcome of SBTs with extraovarian disease. ${ }^{3-7}$ Several histological criteria have been described to identify invasive implants and include findings of a haphazard infiltrative and destructive growth with a predominant stromal component that is characterized as either loose or dense, and the presence of stromal inflammation. ${ }^{3-5}$ A cribriform or micropapillary pattern is another feature of invasive implants and the third feature is the presence of a clear space or cleft around the embedded glands. ${ }^{6,7}$ However, the stromal reaction to invasive implants 
is sometimes weak or absent and tangential sectioning of noninvasive implants on the surface of peritoneal tissue, especially in the omentum, may be mistaken for deep-seated implants so that the criteria described by different investigators can be difficult to apply.

Calretinin is a calcium adhesion protein that is mainly expressed in the nervous system. Calretinin is also expressed in mesothelial cells and their neoplasms, allowing a powerful discriminator of mesothelial cells from other cell types. ${ }^{8,9}$ CD34+ stromal fibrocytes, also referred to as dendritic interstitial cells, show slender cytoplasmic processes that are closely interwoven with those of neighboring fibrocytes, leading to the descriptive term 'CD34+ reticular network.' ${ }^{10,11}$ Such cells occur in large numbers in the connective tissue compartment of various anatomical locations such as the skin, gastrointestinal tract, uterine cervix, breast, pancreas, thyroid, salivary glands, and peripheral nervous system. ${ }^{10-13}$ There is a demonstrable loss of stromal fibrocytes around deposits of invasive carcinoma and this has been shown to be partly accompanied by an increase in $\alpha$-smooth muscle actin positive $(\alpha$-SMA +$)$ myofibroblasts in invasive colorectal adenocarcinoma, ductal adenocarcinoma of the pancreas, ductal breast cancer and squamous cell carcinoma of the uterine cervix. ${ }^{10,12}$

This study examines if a panel of antibodies to calretinin, CD34, and $\alpha$-SMA would be useful for the demonstration of the occurrence and distribution of mesothelial cells, stromal fibrocytes, and myofibroblasts as diagnostic discriminators for invasive and noninvasive peritoneal implants in SBTs of the ovary.

\section{Materials and methods}

\section{Case Selection}

The clinicopathologic records of 227 patients with ovarian SBTs and serous carcinomas collected at the Korea University Hospitals and Samsung Cheil Hospital, Seoul, South Korea between 1994 and 2005 were reviewed. The following data including patient's age, presentation, tumor size, laterality, gross features, presence of implants, stage, treatment, and follow-up were obtained. Surgical treatment was either radical (bilateral salpingooophorectomy with or without hysterectomy), or conservative (conservation of the uterus and at least a portion of one ovary). Additional surgical procedures performed included peritoneal washings, omentectomy, multiple peritoneal biopsies and pelvic and para-aortic lymphadenectomy. A total of 40 cases were selected in which peritoneal biopsies were carried out and paraffin blocks were available. These comprised 20 SBTs with peritoneal implants, 10 serous carcinomas with peritoneal metastasis, and 10 cases of endosalpingiosis.
All slides from the cases were reviewed. The number of hematoxylin and eosin-stained sections ranged from 23 to 41 (mean 33; median 31). The mean number of sections examined per centimeter of the largest tumor dimension available was 1.2. Microscopic features that were evaluated included nuclear atypia, epithelial proliferation, presence of cribriform and micropapillary structures, and presence of invasion in the primary tumors and implants. SBTs were classified as 'typical SBT' or 'SBT with micropapillary and/or cribriform pattern' according to previously published criteria. ${ }^{14-19}$ Briefly, tumors characterized by variably sized fibrous papillae with a hierarchical branching architecture and lined by mildly to moderately atypical columnar, polygonal, or hobnail cells with tufting and detachment of epithelial clusters were classified as 'typical SBT.' Tumors displaying proliferation of elongate filiform micropapillae with little or no fibrovascular cores or those displaying a fused or cribriform pattern were classified as 'SBT with micropapillary/cribriform pattern.' The micropapillae were at least five times longer than wide. At least one contiguous focus of micropapillary architecture measuring $5 \mathrm{~mm}$ in diameter was required for a diagnosis of SBT with micropapillary/cribriform pattern. The peritoneal implants were classified as 'invasive' or 'noninvasive' using the criteria of Bell et al. ${ }^{5,6}$ Briefly, the occurrence of any one of three criteria were used for the diagnosis of 'invasion': (1) haphazard invasion of normal underlying tissue, (2) presence of a cribriform/micropapillary architecture, or (3) solid epithelial nests or small papillae separated from the surrounding stroma by a cleft or space. Implants displaying any one of these features were classified as 'invasive.' Implants lacking all three features were considered 'noninvasive.' Seven cases with invasive implants also contained areas of noninvasive implants. These cases of mixed invasive and noninvasive implants were designated 'SBTs with invasive implants.' Some implants were accompanied by a marked stromal reaction with fibrin deposition which was associated with hemorrhage, inflammation, edema, necrosis and/or fibrosis, and loosely adherent to the subjacent peritoneum, having a plaque-like appearance. They comprised small glands, papillae, glomerular structures, solid nests or single cells with or without surrounding clefts which separated the epithelium from stroma. They did not show destructive invasion. These were classified 'noninvasive desmoplastic implants' ${ }^{5}$ even though they were surrounded by a cleft or space.

In all, 4-25 areas containing invasive or noninvasive implants, or metastatic nests from each case were selected and evaluated for mesothelial cells, stromal fibrocytes, and myofibroblasts. The total foci/nests assessed were 40 nests from 10 cases of endosalpingiosis, 100 metastatic nests from 10 serous carcinomas, 60 noninvasive nests from 13 SBTs with only noninvasive implants, and 140 nests 
from seven SBTs with mixed invasive and noninvasive implants. Among the latter 140 nests, 100 showed the histologic features of invasive implants, the remaining being noninvasive.

\section{Immunohistochemical Study}

Immunohistochemical studies were performed on formalin-fixed, paraffin-embedded $5-\mu \mathrm{m}$ sections from all patients. Three consecutive sections were prepared from each tissue block and stained for the following markers: calretinin for mesothelial cells, CD34 for stromal fibrocytes, and $\alpha$-SMA for myofibroblasts. The sections were deparaffinized in xylene and rehydrated through graded alcohols. Antigen retrieval was performed in $1 \mathrm{mmol} / \mathrm{l}$ of EDTA (pH 8.0) in a microwave oven at $98^{\circ} \mathrm{C}$. Endogenous peroxidases were inactivated by immersing the sections in $0.3 \%$ hydrogen peroxide for 20 min. Primary antibodies used were calretinin (DAK Calret, dilution 1:200; Dako, Carpinteria, CA, USA), CD34 (QBEN/10, dilution 1:200; Immunotech, Marseilles, France), and $\alpha$-SMA (IA4, dilution 1:200; BioGene, San Ramon, CA, USA). Staining was performed with the DAKO EnVision kit (DAKO) and the sections were developed with 3,3'-diaminobenzidine tetrahydrochloride and counterstained with hematoxylin.

\section{Statistical Analysis}

Differences in the positive rates for calretinin, CD34, and $\alpha$-SMA between invasive and noninvasive implants were assessed by $\chi^{2}$ test for categorical variables. A $P$-value of $<0.01$ was considered to be significant.

\section{Results}

\section{Clinicopathologic Findings (Tables 1 and 2)}

In all, 13 (65\%) SBTs had only noninvasive implants and seven $(35 \%)$ had both invasive and noninvasive implants. The clinicopathologic features are shown in Tables 1 and 2. All SBTs with invasive implants were stage III $(100 \%)$, and of the 13 with noninvasive implants eight $(62 \%)$ were stage II, and five $(38 \%)$ were stage III. Invasive implants occurred in younger patients (median 33 years) compared to those with noninvasive implants (median 37 years), and SBTs with invasive implants were more often bilateral tumors (7/7) and exophytic or mixed exophytic and intracystic papillary tumors (7/7) compared to SBTs with noninvasive implants (8/13 and $9 / 13$, respectively). They more frequently showed the micropapillary/cribriform pattern $(4 / 7)$ compared to SBTs with noninvasive implants $(2 / 13)$ (Table 2).

\section{Immunohistochemical Results (Tables 3 and 4)}

Nonlesional peritoneal tissue and omentum in SBTs and serous carcinomas

All nonlesional peritoneal tissues including subserosa of uterus, salpinx, ovary, ligament, colon, appendix, recto-vaginal cul de sac, urinary bladder,

Table 1 Clinicopathologic features of SBTs with peritoneal implants

\begin{tabular}{|c|c|c|c|c|c|c|c|}
\hline Case no. & Age (years) & $\begin{array}{c}\text { Tumor size }(\mathrm{cm}), \\
\text { left/right }\end{array}$ & Gross findings & $M P / C$ pattern & Type of implant & Stage & Implant sites \\
\hline 1 & 32 & $18 / 24$ & Intracystic & Absent & NID,NIE & 2 & B,C,P \\
\hline 2 & 37 & $5 / 6.5$ & Exophytic & Absent & NID,NIE & 2 & S \\
\hline 3 & 56 & $-/ 5$ & Mixed & Absent & NIE & 2 & $\mathrm{O}, \mathrm{P}$ \\
\hline 4 & 32 & $-/ 8$ & Mixed & Absent & NID & 3 & $\mathrm{C}, \mathrm{LN}, \mathrm{O}, \mathrm{P}$ \\
\hline 5 & 68 & $5 / 11$ & Mixed & Present & I & 3 & $P, \mathrm{C}, \mathrm{R}, \mathrm{U}$ \\
\hline 6 & 31 & $20 / 9$ & Intracystic & Present & NIE & 3 & $\mathrm{C}, \mathrm{OM}, \mathrm{R}$ \\
\hline 7 & 53 & 9/- & Mixed & Absent & NIE & 2 & $\mathrm{O}$ \\
\hline 8 & 32 & $13 /-$ & Intracystic & Absent & NID & 2 & $\mathrm{C}$ \\
\hline 9 & 31 & $15 / 5$ & Mixed & Absent & I & 3 & $O M$ \\
\hline 10 & 40 & $5 / 11$ & Intracystic & Absent & NIE & 2 & U \\
\hline 11 & 34 & $-/ 9$ & Exophytic & Absent & NID & 3 & $\mathrm{C}, \mathrm{OM}$ \\
\hline 12 & 51 & $9 / 11$ & Mixed & Present & I & 3 & $S, \mathrm{~A}, \mathrm{~B}, \mathrm{C}, \mathrm{OM}, \mathrm{P}, \mathrm{R}$ \\
\hline 13 & 38 & $5 / 12$ & Mixed & Absent & NID,NIE & 3 & U,LN \\
\hline 14 & 56 & $6.5 / 7$ & Exophytic & Absent & NIE & 3 & OM \\
\hline 15 & 33 & $13 / 11$ & Mixed & Present & I & 3 & $O M, \mathrm{~S}, \mathrm{U}$ \\
\hline 16 & 27 & $5 / 5$ & Exophytic & Present & I & 3 & $O M, S, U, \mathrm{C}, \mathrm{LN}$ \\
\hline 17 & 67 & $5 / 12$ & Mixed & Absent & NID,NIE & 2 & R,U \\
\hline 18 & 33 & $20 / 11$ & Mixed & Absent & I & 3 & $O M$ \\
\hline 19 & 39 & $14 / 6$ & Mixed & Absent & I & 3 & $O M$ \\
\hline 20 & 31 & $9 / 6$ & Mixed & Present & NIE & 2 & S \\
\hline
\end{tabular}

SBTs = serous borderline tumors; $\mathrm{MP}=$ micropapillary; $\mathrm{C}=$ cribriform; $\mathrm{I}=$ invasive; $\mathrm{NID}=$ noninvasive desmoplastic; $\mathrm{NIE}=$ noninvasive epithelial; $\mathrm{Ab}=$ primary tumor not identified; $\mathrm{A}=$ appendix; $\mathrm{B}=$ bladder; $\mathrm{C}=$ cul de sac; $\mathrm{L}=$ ligament; $\mathrm{LN}=$ lymph node; $\mathrm{O}=\mathrm{ovary}$; $\mathrm{OM}=$ omentum; $\mathrm{P}=$ peritoneum; $\mathrm{R}=$ rectum; $\mathrm{S}=$ salpinx; $\mathrm{U}=$ uterus.

Italics = invasive implant in patients with mixed invasive and noninvasive implants. 
and omentum contained large numbers of CD34+ fibrocytes, which formed a reticular network in the connective tissue stroma. CD34 immunostaining also highlighted abundant fibrocytes in the myometrium and the muscle layers of the intestine and urinary bladder. Fibrocytes were generally evenly distributed throughout the stroma with condensation around vessels. CD34 expression in these cells was strong with staining of cytoplasm and cell membrane. In inflamed or fibrotic tissues, stromal fibrocytes were not present. Mesothelial cells lining

Table 2 Clinicopathologic findings in SBTs with invasive and noninvasive peritoneal implants

\begin{tabular}{|c|c|c|c|}
\hline & $\begin{array}{c}\text { Total } \\
(\mathrm{n}=20)\end{array}$ & $\begin{array}{c}\text { Invasive } \\
\text { implants } \\
(\mathrm{n}=7)(\%)\end{array}$ & $\begin{array}{c}\text { Noninvasive } \\
\text { implants } \\
(\mathrm{n}=13)(\%)\end{array}$ \\
\hline \multicolumn{4}{|l|}{ Laterality } \\
\hline Unilateral & 5 & $0(0)$ & $5(100)$ \\
\hline Bilateral & 15 & $7(47)$ & $8(53)$ \\
\hline \multicolumn{4}{|l|}{ Gross findings } \\
\hline Intracystic & 4 & $0(0)$ & $4(100)$ \\
\hline Exophytic & 4 & $1(25)$ & $3(75)$ \\
\hline Mixed & 12 & $6(50)$ & $6(50)$ \\
\hline \multicolumn{4}{|l|}{ Histologic type } \\
\hline $\begin{array}{l}\text { Micropapillary/ } \\
\text { cribriform }\end{array}$ & 6 & $4(67)$ & $2(33)$ \\
\hline Typical & 14 & $3(21)$ & $11(79)$ \\
\hline \multicolumn{4}{|l|}{ Stage } \\
\hline 1 & 0 & $0(0)$ & $0(0)$ \\
\hline 2 & 8 & $0(0)$ & $8(100)$ \\
\hline 3 & 12 & $7(58)$ & $5(42)$ \\
\hline 4 & 0 & $0(0)$ & $0(0)$ \\
\hline $\begin{array}{l}\text { Median tumor size } \\
\text { (cm) (range) }\end{array}$ & & $11(5-20)$ & $9(5-24)$ \\
\hline $\begin{array}{l}\text { Median age at } \\
\text { presentation } \\
\text { (range) }\end{array}$ & & $33(27-68)$ & $37(31-67)$ \\
\hline
\end{tabular}

the peritoneum were well preserved and showed strong positive reaction for calretinin. The occurrence of $\alpha$-SMA + myofibroblasts was not found in all sections of nonlesional peritoneal tissue and omentum examined but they were increased in areas of inflammation and fibrosis.

\section{Endosalpingiosis}

The glandular nests of endosalpingiosis were superficially embedded in the peritoneal tissues overlying the uterus, salpinx, ovary, colon, appendix, and omentum. They comprised small simple glands or papillae, the latter projecting into a clear space, which was lined by a single layer of cuboidal or ciliated tubal cells. They were smooth contoured and not associated with destructive invasion or desmoplastic response. All 40 such nests, with the exception of three that occurred in inflamed tissue, showed well-preserved stromal fibrocytes that were sharply condensed around the glandular nests (Figure 1a). The epithelial cells lining the clear space in all 40 nests did not label with calretinin

Table $4 \alpha$-SMA+ myofibroblasts in peritoneal implants of SBTs and metastasis from serous carcinomas

\begin{tabular}{lcc}
\hline & $\alpha-S M A(+)$ & $\alpha-S M A(-)$ \\
\hline $\begin{array}{l}\text { Noninvasive implants }(\mathrm{n}=100) \\
\text { Epithelial }(\mathrm{n}=50) \\
\quad \begin{array}{l}\text { SBTs with noninvasive implants } \\
(n=40)\end{array}\end{array}$ & 3 & 37 \\
$\quad$ SBTs with mixed implants $(n=10)$ & 1 & 9 \\
$\begin{array}{l}\text { Desmoplastic }(\mathrm{n}=50) \\
\quad \text { SBTs with noninvasive implants } \\
\quad(n=20)\end{array}$ & 20 & 0 \\
$\quad \begin{array}{l}\text { SBTs with mixed implants }(n=30) \\
\text { Invasive implants }(n=100)\end{array}$ & 30 & 0 \\
$\quad$ Metastatic nests $(n=100)$ & 100 & 0 \\
\hline
\end{tabular}

Table 3 Calretinin and CD34 staining in peritoneal implants of SBTs, metastatic nests of serous carcinomas, and endosalpingiosis

\begin{tabular}{|c|c|c|c|c|}
\hline & \multicolumn{2}{|c|}{ Calretinin (+) } & \multicolumn{2}{|c|}{ Calretinin $(-)$} \\
\hline & $C D 34(+)$ & $C D 34(-)$ & CD $34(+)$ & $C D 34(-)$ \\
\hline Endosalpingiosis $(n=40)$ & 0 & 0 & 37 & 3 \\
\hline \multicolumn{5}{|l|}{ Noninvasive implants $(\mathrm{n}=100)$} \\
\hline SBTs with noninvasive implants $(n=40)$ & 37 & 2 & 1 & 0 \\
\hline SBTs with mixed implants $(n=10)$ & 10 & 0 & 0 & 0 \\
\hline \multicolumn{5}{|l|}{ Desmoplastic $(\mathrm{n}=50)$} \\
\hline SBTs with noninvasive implants $(n=20)$ & 0 & 1 & 0 & 19 \\
\hline SBTs with mixed implants $(n=30)$ & 12 & 10 & 8 & 0 \\
\hline Invasive implants $(n=100)$ & 0 & 0 & 0 & 100 \\
\hline Metastatic nests $(n=100)$ & 0 & 0 & 0 & 100 \\
\hline
\end{tabular}



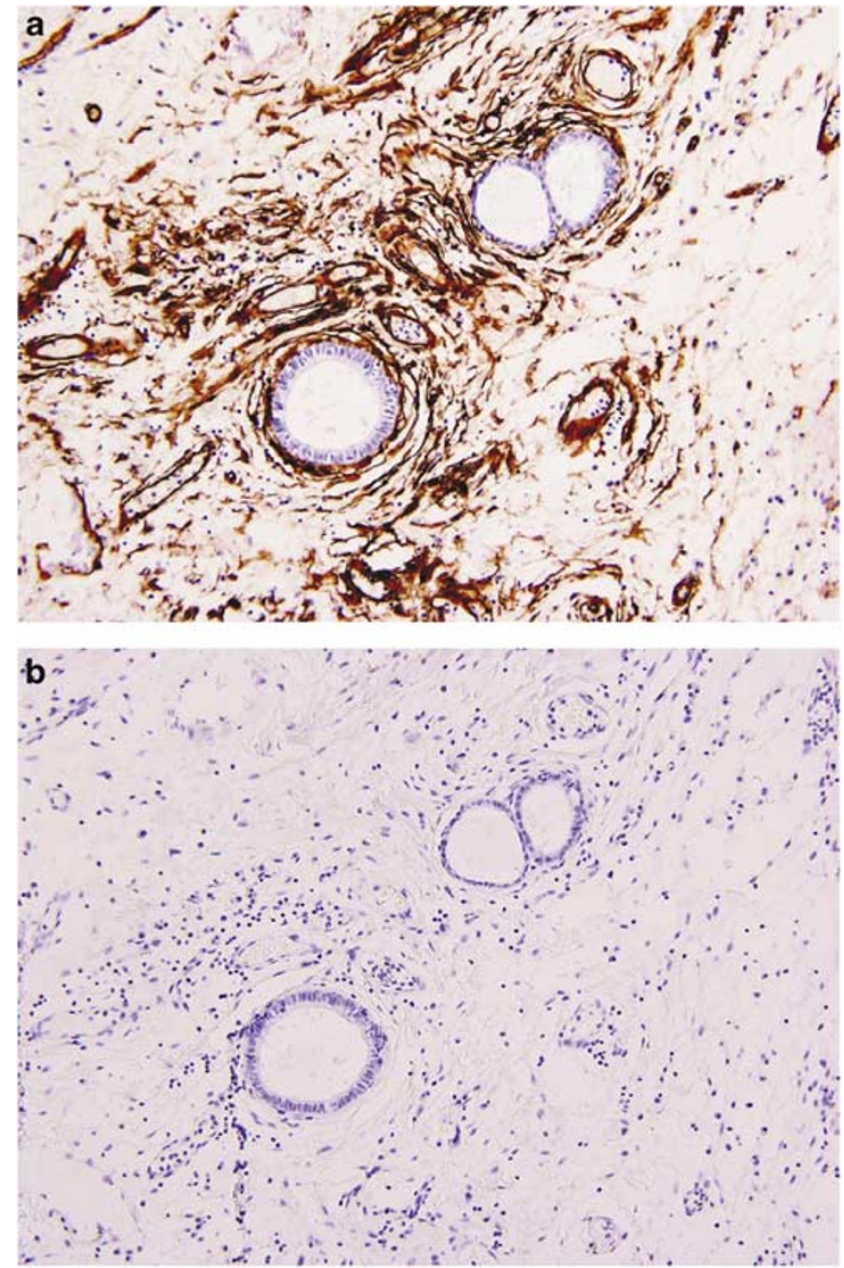

Figure 1 Endosalpingiosis in the omentum. CD34 + fibrocytes are distributed throughout the stroma with condensation around the epithelial inclusions and vessels (a). Calretinin does not label the epithelial inclusions (b).

(Figure 1b). Myofibroblasts were not present around such deposits of endosalpingiosis.

\section{Metastatic nests in serous carcinoma}

Metastatic ovarian serous carcinomas showed numerous large solid epithelial nests, glands, and papillae. They were deeply and irregularly infiltrating and haphazardly distributed within a desmoplastic stroma. Many nests were surrounded by clefts that separated the epithelium from stroma. The tumor cells showed marked cytologic atypia compared to the tumor cells of SBTs, which displayed mild to moderate atypia. All 100 metastatic deposits examined showed no staining with calretinin and a loss of the normal stromal fibrocytes around the metastatic nests (Figure 2a). The occurrence of $\alpha$-SMA + myofibroblasts was present in all 100 nests (Figure 2b).

\section{Nests in SBTs with only noninvasive implants}

The nests in SBTs with only noninvasive implants were mostly exophytic or superficially embedded
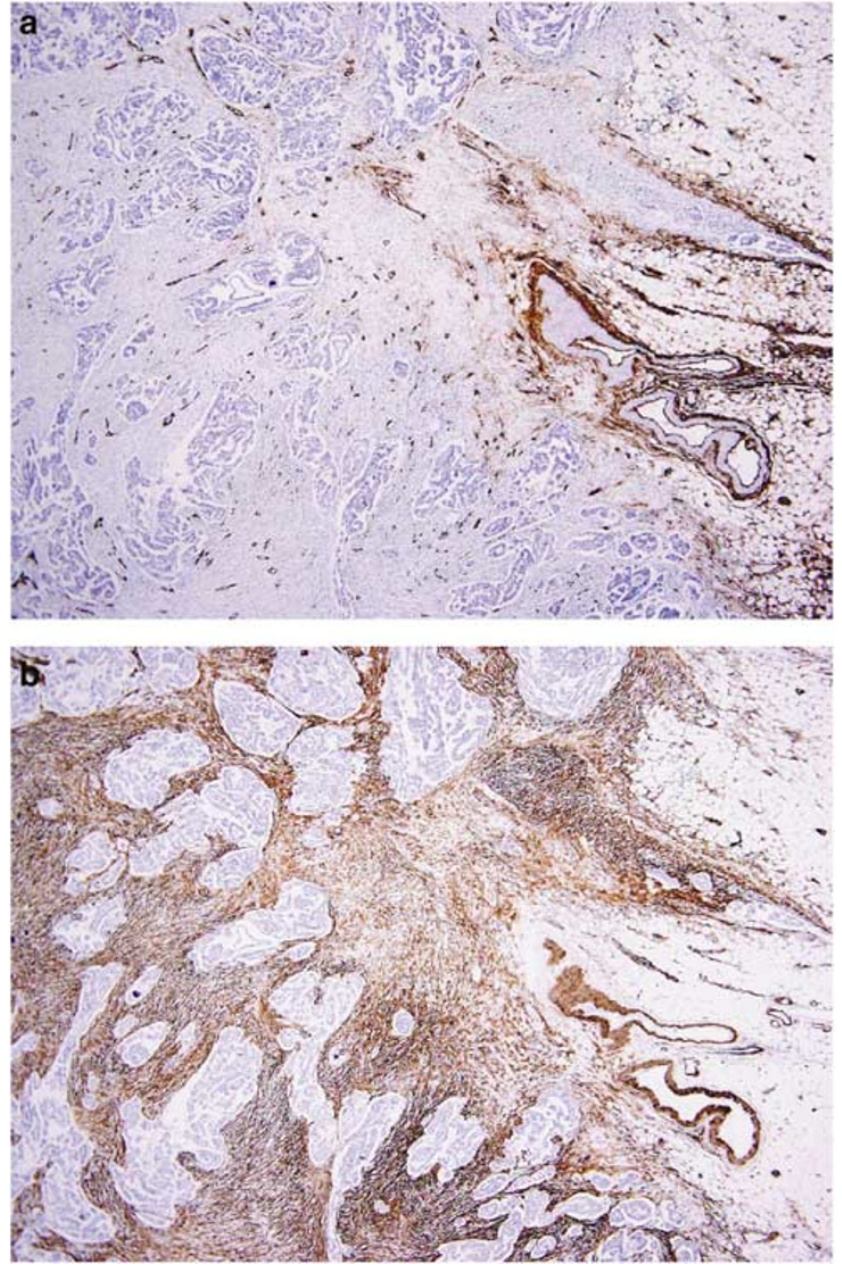

Figure 2 Metastatic serous carcinoma in the omentum comprising numerous large solid epithelial nests, glands, and papillae, which are deeply infiltrating and haphazardly distributed within a desmoplastic stroma. There is loss of stromal CD34 + fibrocytes (a) and occurrence of $\alpha$-SMA + myofibroblasts around the metastatic nests (b).

in the peritoneal tissue and omentum. Some nests were deeply seated with absent or a weak stromal reaction. The exophytic implants comprised large or small branching papillae sharply demarcated from the stroma. Most of the superficially or deeply embedded noninvasive implants showed a glomerular appearance with papillae projecting into a clear space that was lined by a single layer of cells that were flat to cuboidal with small, regular and hyperchromatic nuclei in contrast to the tumor cells of papillae, which harbored large, irregular and hyperchromatic nuclei. The clear space encasing the glomerular nests was differentiated from the clefts of invasive implants by the presence of a cellular lining. All noninvasive epithelial implants were smooth contoured with neither destructive invasion nor desmoplastic stromal reaction around the implants. Stromal CD34+ fibrocytes were well preserved in 38/40 nests studied, with smooth and well-demarcated borders 

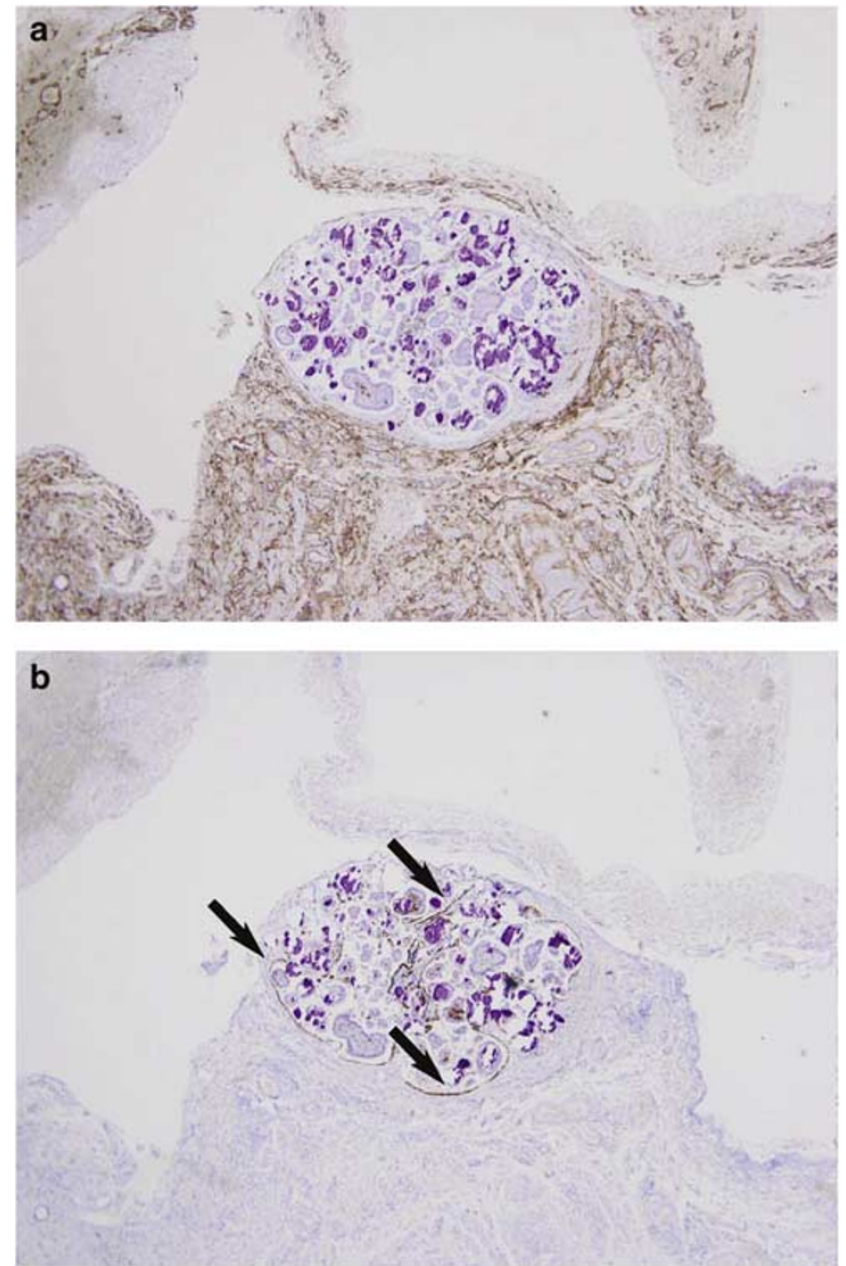

Figure 3 Noninvasive epithelial implant superficially embedded in the subserosa of uterus. The small papillae project into a clear space which is lined by a single layer of flat mesothelial cells, producing a glomerular appearance. Stromal CD34 + fibrocytes are well preserved with a sharp border around the implants formed by condensation of fibrocytes (a). The mesothelial cells lining the clear space show a positive reaction for calretinin (arrows) (b).

(Figure 3a). Calretinin showed strong positive reaction to the mesothelial cells lining the clear space in 39/40 nests, whereas the tumor cells of the papillae did not (Figure $3 \mathrm{~b}$ ). $\alpha$-SMA + myofibroblasts were not present around 37/40 noninvasive nests.

The 'noninvasive desmoplastic implants'5 comprised small glands, papillae, glomerular structures, solid nests, or single cells with or without surrounding clefts which separated the epithelium from stroma (Figure 4a). They did not show destructive invasion. In such desmoplastic plaques, there were no stromal CD34 + fibrocytes around all 20 evaluated nests (Figure 4b) and mesothelial cells were not present in 19/20 nests. The stroma of the surface plaques resembled granulation tissue and $\alpha$-SMA + myofibroblasts were present around all 20 nests.
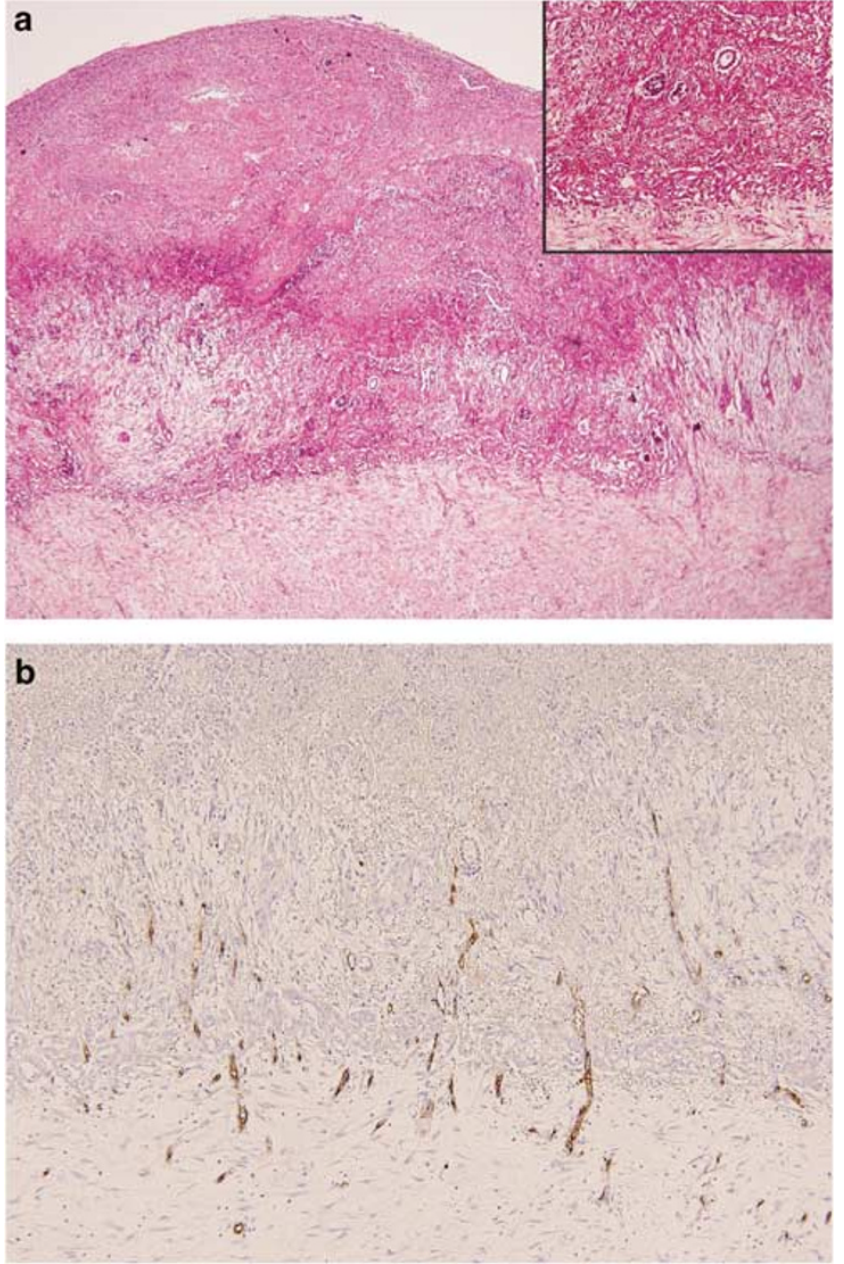

Figure 4 Plaque-like noninvasive desmoplastic implants in granulation tissue overlying the uterus. Small glands, papillae, solid nests, or single cells are embedded in loosely textured edematous and hemorrhagic stroma with the surrounding clefts which separated the epithelium from the stroma (a). Stromal CD34 + fibrocytes are not present in the granulation tissue with blood vessels showing a positive reaction for CD34 (b).

\section{Nests in SBTs with mixed implants}

The nests in SBTs with both invasive and noninvasive peritoneal implants were solid, glandular, papillary, micropapillary, cribriform, and glomerular. Some implants, especially solid or micropapillary nests were surrounded by clefts that separated the epithelium from the stroma. These were deeply and irregularly infiltrating within a desmoplastic stroma. Such invasive implants consisted entirely of tumor cells with large, irregular, and hyperchromatic nuclei. Calretinin + mesothelial cells were not present in all 100 evaluated nests (Figure 5a). Furthermore, all invasive nests showed loss of surrounding stromal CD34 + fibrocytes (Figure 5b) with the occurrence of $\alpha$-SMA + myofibroblasts around all 100 invasive nests (Figure 6).

Some invasive implants showed a glomerular appearance with an encasing space lined by tumor 
370
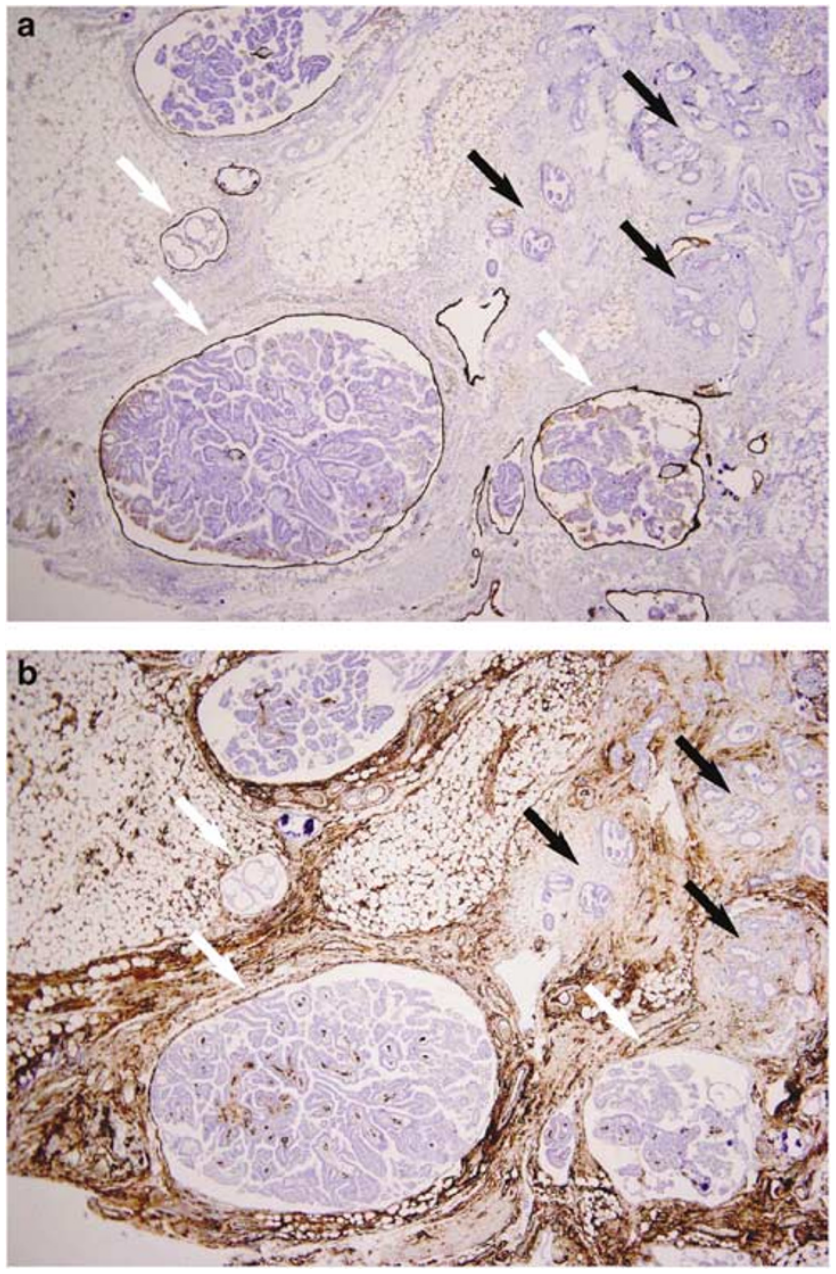

Figure 5 Mixed invasive and noninvasive implants deeply embedded in the omentum. The noninvasive glomerular nests (white arrows) show calretinin + mesothelial cells (a) and stromal CD34 + fibrocytes around the nests (b). Invasive nests including some glomerular nests (black arrows) show loss of calretinin + mesothelial cells (a) and stromal CD $34+$ fibrocytes (b).

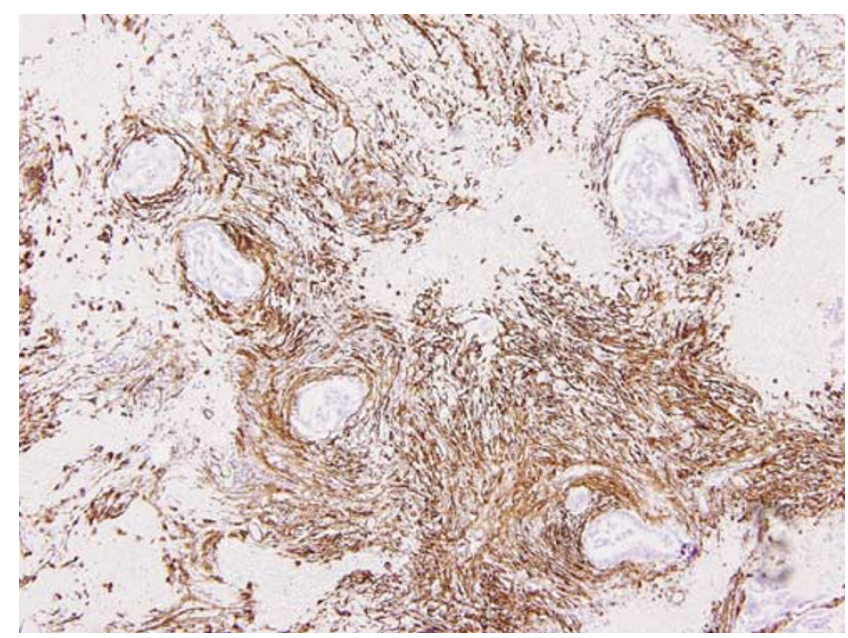

Figure $6 \alpha$-SMA staining reveals the presence of myofibroblasts around invasive implants. cells, which were similar to those of the papillae with large, irregular, and hyperchromatic nuclei. These cells did not label with calretinin. These invasive implants were both smooth or irregularly contoured and deeply seated in the peritoneal tissue. This contrasted with noninvasive glomerular nests which were often superficially located, had smooth contours and were lined by calretinin + mesothelial cells. Some invasive nests showed irregular or jagged outlines with marked and confluent proliferation of neoplastic cells that created a maze-like or cribriform pattern. They were frequently associated with a desmoplastic reaction in the surrounding stroma and all showed loss of stromal CD34 + fibrocytes.

Many noninvasive nests with or without a mesothelial cell component were adjacent to or juxtaposed to invasive implants. They were smooth contoured and superficially or deeply located in the peritoneal tissues. Some were found in desmoplastic stroma which was induced by the adjacent invasive nests. We interpreted such lesions as noninvasive desmoplastic implants. They retained an encasing layer of calretinin + mesothelial cells in 22/30 lesions (Figure 5a). Stromal CD34 + fibrocytes were preserved in 20/30 lesions (Figure 5b).

The noninvasive epithelial implants present in SBTs with mixed implants showed findings similar to those cases with purely noninvasive implants.

\section{Statistical Analysis}

There were significant differences $(P<0.01)$ in the positive rates for calretinin, CD34, and $\alpha$-SMA between the invasive and noninvasive groups. A total of $100 \%(100 / 100)$ of invasive and $28 \%$ (28/ 100) of noninvasive nests showed absence of calretinin + mesothelial cells. The sensitivity and specificity of loss of calretinin in the identification of invasive nests were 100 and $72 \%$, respectively. A total of $100 \%(100 / 100)$ of invasive and $32 \%(32 /$ 100) of noninvasive nests showed loss of CD34+ fibrocytes around the nests. The sensitivity and specificity of loss of CD34+ fibrocytes in the identification of invasive nests were 100 and $68 \%$, respectively. A total of $100 \%(100 / 100)$ of invasive nests and $54 \%(54 / 100)$ of noninvasive nests showed occurrence of $\alpha$-SMA + myofibroblasts. The sensitivity and specificity of $\alpha$-SMA + myofibroblasts in the identification of invasive nests were 100 and $46 \%$, respectively. When all three antibodies were combined, the sensitivity was $100 \%$ and specificity $81 \%$. The false-positive and falsenegative rates for loss of calretinin + mesothelial cells in the identification of invasive nests were 0 and $28 \%$, respectively. The false-positive and falsenegative rates for loss of CD34 + fibrocytes in the identification of invasive nests were 0 and $32 \%$, respectively. The false-positive and false-negative rates for $\alpha$-SMA + myofibroblasts in the identification of invasive nests were 0 and $54 \%$, respectively. 


\section{Discussion}

The introduction of the term 'SBTs constituted a major advancement in classification of ovarian serous tumors because it specifically separated a group with very different clinical behavior and prognosis to that of conventional serous carcinomas. The adopted definitions noted that these tumors have histologic features such as epithelial proliferation and atypia that fall between those of serous cystadenoma and serous cystadenocarcinoma but lack the destructive infiltration of the latter. Stage is a powerful predictor of outcome and recurrence is more frequently associated with high-stage disease. It was also recognized that the invasive peritoneal lesions are associated with adverse prognosis ${ }^{3,6}$ and oncologists believe that patients with noninvasive implants do not benefit from chemotherapy. ${ }^{6}$ For effective management of patients with invasive and noninvasive implants, agreement must be reached on the criteria for their diagnosis.

Several histological criteria have been described to identify invasive implants. Some studies suggest that the only valid criterion for invasion is destructive invasion of underlying normal tissue and the only feature specifically associated with a poor outcome was obvious destructive invasion of the underlying tissue. ${ }^{4,15}$ Others suggest that besides destructive invasion of the underlying normal tissue, the additional findings of a micropapillary architecture and solid epithelial nests or small papillae surrounded by clefts, are highly characteristic of invasive implants. They revealed that after a mean follow-up of 7.4 years, the survival of patients with noninvasive peritoneal implants was $95.3 \%$ as compared with $66 \%$ for invasive implants, ${ }^{7}$ making these additional features important prognostic parameters in SBTs. An additional problem of the criteria with the interpretation of peritoneal implants is the fact that distinguishing between invasive and noninvasive implants depends predominantly on the evaluation of the junction between the implant and surrounding normal tissue, which is often not available in small biopsy specimens. ${ }^{18}$ We reveal that the use of three markers to identify mesothelial cells, stromal fibrocytes, and myofibroblasts is valuable for evaluation of invasiveness of each peritoneal nest and can be available in small biopsy specimens. Analysis of the published series reveals that, regardless of probable differences in diagnostic criteria used, the recurrence rate is higher and the long-term survival rate lower in cases with invasive implants, regardless of whether the ovarian tumor does or does not exhibit micropapillary features. Thus, if micropapillary SBTs do indeed have a worse prognosis, it is probably because of their greater likelihood of being associated with invasive extraovarian disease when compared with typical SBTs. ${ }^{18}$ To exclude the noninvasive nests with micropapillary pattern, nests with micropapillary pattern which were accompanied with irregular or infiltrative borders within the peritoneal tissue were selected as invasive nests in this study. All examined invasive implants including nests with clefts and the micropapillary/cribriform pattern showed loss of calretinin + mesothelial cells and CD34 + fibrocytes and occurrence of SMA + myofibroblasts (Tables 3 and 4). However, other nests with micropapillary pattern and smooth contour showed preserved calretinin + mesothelial cells and/or CD34 + stromal fibrocytes such as noninvasive implants.

It is also important to note that implants are heterogeneous, and various types may coexist in different areas. Therefore, sampling of as many implants as possible is recommended. Our study reveals that omentum is the most frequent site for the invasive implants. Even when other peritoneal tissues have no implants or only noninvasive implants, the omentum may contain invasive implants and sufficient omentum must be adequately sampled and examined for invasive implants.

Calretinin labeled the mesothelial cells that appeared to line the spaces around glomerular noninvasive implants with a sensitivity of $100 \%$ and specificity of $72 \%$. Calretinin was not immunoexpressed in the tumor cells. Such nests, although appearing deeply seated, are likely to represent deep invaginations of surface implants that carry with them surface mesothelium or alternatively, they are tangential cuts of more superficial invaginations.

Most noninvasive epithelial implants displayed a glomerular appearance with smooth contours, sharp demarcation from the surrounding stroma, and were not associated with stromal clefts. Stromal CD34+ fibrocytes around noninvasive implants were preserved whereas there was disruption and loss of the normal distribution of stromal fibrocytes around invasive implants, with a sensitivity of $100 \%$ and specificity of $68 \%$.

$\alpha$-SMA + myofibroblasts when present around epithelial implants indicated stromal desmoplasia with $100 \%$ sensitivity. However, specificity was only $46 \%$ as a stromal response was also seen with noninvasive desmoplastic implants. Such noninvasive implants were plaque like and formed of fibrosis and granulation tissue that entrapped gland-like structures. While such epithelial implants may often resemble mesothelial inclusions, they can also be papillary and have cells similar to those of SBTs; however, the stromal component is frequently disproportionately abundant and depending on the stage of evolution of the lesion may also show necrosis and hemorrhage characteristic of superficial desmoplastic noninvasive implants. ${ }^{3,6,15}$

The other situation in which stromal desmoplasia was seen in association with noninvasive implants was in those cases of SBTs with mixed implants. Such noninvasive desmoplastic implants are different from the noninvasive desmoplastic implants in granulation tissue described above. This represents 
a second type of noninvasive implant in which a desmoplastic response may be seen and the presence of desmoplasia should not be mistaken for a feature of invasion in this setting. The desmoplastic response to invasion is frequently so extensive that it encompasses the stroma around adjacent noninvasive implants so that myofibroblasts are associated with such noninvasive nests.

The presence of stromal clefts around epithelial nests has been described as a feature of invasive implants. ${ }^{6}$ However, we observed such clefts around the glands of noninvasive desmoplastic implants. The histogenesis of such clefts in invasive and noninvasive desmoplastic implants may well be related to the presence of myofibroblasts that may exert contractile forces that cause the separation of stroma from epithelium to produce the cleft. To our knowledge, the relation of stromal fibrocytes to myofibroblasts is not fully understood. It is speculative that the stromal fibrocytes may have a precursor relationship to myofibroblasts and this has yet to be shown.

We show, for the first time, that the use of three markers to identify mesothelial cells, stromal fibrocytes, and myofibroblasts is a valuable adjunct to morphological diagnosis of invasive implants in SBTs. The combined use of these three markers has a sensitivity and specificity of 100 and $81 \%$, respectively. Although these methods may not be helpful in noninvasive desmoplastic implants, they should be employed especially when small biopsies of the peritoneal tissue are submitted for evaluation of invasiveness of implants in SBTs.

\section{References}

1 International Federation of Gynecology and Obstetrics. Classification and staging of malignant tumors in the female pelvis. Acta Obstet Gynecol Scand 1971;50:1-7.

2 Serov SF, Scully RE, Sobin LH. Histological Typing of Ovarian Tumors: WHO International Histological Classification of Tumors, no. 9. Springer: Heidelberg, 1973.

3 Bell DA, Weinstock MA, Scully RE. Peritoneal implants of ovarian serous borderline tumors: histologic features and prognosis. Cancer 1988;62:2212-2222.

4 Prat J, De Nictolis M. Serous borderline tumors of the ovary: a long-term follow-up study of 137 cases, including 18 with a micropapillary pattern and 20 with microinvasion. Am J Surg Pathol 2002;26:1111-1128.

5 Bell DA, Longacre TA, Prat J, et al. Serous borderline (low malignant potential, atypical proliferative) ovarian tumors: workshop perspectives. Hum Pathol 2004;35:934-948.

6 Bell KA, Smith Sehdev AE, Kurman RJ. Refined diagnostic criteria for implants associated with ovarian atypical proliferative serous tumors (borderline) and micropapillary serous carcinomas. Am J Surg Pathol 2001;25:419-432.

7 Seidman JD, Kurman RJ. Ovarian serous borderline tumors: a critical review of the literature with emphasis on prognostic indicators. Hum Pathol 2000;31:539-557.

8 Doglioni C, Tos AP, Laurino L, et al. Calretinin: a novel immunocytochemical marker for mesothelioma. Am J Surg Pathol 1996;20:1037-1046.

9 Gotzos V, Vogt P, Celio MR. The calcium binding protein calretinin is a selective marker for malignant pleural mesotheliomas of the epithelial type. Pathol Res Pract 1996;192:137-147.

10 Barth PJ, Ramaswamy A, Moll R. CD34 ${ }^{+}$fibrocytes in normal cervical stroma, cervical intraepithelial neoplasia III, and invasive squamous cell carcinoma of the cervix uteri. Virchows Arch 2002;441: 564-568.

11 Suster S, Fisher C. Immunoreactivity for the human hematopoietic progenitor cell antigen (CD34) in lipomatous tumors. Am J Surg Pathol 1997;21:195-200.

12 Nakayama H, Enzan H, Miyazaki E, et al. CD34 positive stromal cells in gastric adenocarcinomas. J Clin Pathol 2001;54:846-848.

13 Khalifa MA, Montgomery EA, Ismiil N, et al. What are CD34+ cells in benign peripheral nerve sheath tumors? Am J Clin Pathol 2000;114:123-126.

14 Burks RT, Sherman ME, Kurman RJ. Micropapillary serous carcinoma of the ovary. A distinctive low-grade carcinoma related to serous borderline tumors. Am J Surg Pathol 1996;20:1319-1330.

15 Eichhorn JH, Bell DA, Young RH, et al. Ovarian serous borderline tumors with micropapillary and cribriform patterns. A study of 40 cases and comparison with 44 cases without these patterns. Am J Surg Pathol 1999; 23:397-409.

16 Seidman JD, Kurman RJ. Subclassification of serous borderline tumors of the ovary into benign and malignant types. A clinicopathologic study of 65 advanced stage cases. Am J Surg Pathol 1996;20: 1331-1345.

17 Silva EG, Kurman RJ, Russell P, et al. Symposium: ovarian tumors of borderline malignancy. Int J Gynecol Pathol 1996;15:281-302.

18 Silverberg SG, Bell DA, Kurman RJ, et al. Borderline ovarian tumors: key points and workshop summary. Hum Pathol 2004;35:910-917.

19 Sherman ME, Berman J, Birrer MJ, et al. Borderline ovarian tumors: diverse contemporary viewpoints on terminology and diagnostic criteria with illustrative images. Hum Pathol 2004;35:918-933. 